\title{
Recursive Differential Systems in Nonlinear Mechanics
}

\author{
Claire David ${ }^{\dagger *}$, Marine Marcilhac and Alain Rigolot ${ }^{\dagger}$ \\ Université Pierre et Marie Curie-Paris 6 \\ † Institut Jean Le Rond d'Alembert, UMR CNRS 7190, \\ Boîte courrier $n^{0} 162$, 4 place Jussieu, 75252 Paris, cedex 05, France
}

\begin{abstract}
The Classical Strength of Materials for beams is represented through the first two terms of the asymptotic expansion of the solution of Navier's equations. The method of asymptotic expansions with respect to the inverse of the slenderness of the beam permits us to obtain an approximate solution of Saint-Venant's problem. For the elasticity of the second order, the displacement field is obtained as the sum of a series, the general term of which at the $n^{\text {th }}$ order is the solution of a differential recursive system. We presently propose a general way of solving this kind of systems. The exact solution is given explicitly in the case of a slender field (beam).
\end{abstract}

Keywords: asymptotic developments, differential recursive systems..

\section{Introduction}

Asymptotic expansion methods enables us to build the successive terms of a formal power series expansion of the solution of a specific problem. While this technique is commonly applied to linear problems within the context of classical elasticity, it is also used to study nonlinear problems.

The asymptotic approach was first used by Hay [5], who determined the first terms of St Venant's problem, for a curvilinear body. Other kinds of problems, for straight beams, have been studied by Muller [6]. Antman [7] made a complete study of nonlinear curvilinear fields.

The asymptotic approach was then used by A. Rigolot [1] for second-order elasticity to determine the equilibrium conditions of a straight beam submitted to a lateral loading. He was the first to extend asymptotic methods to non-linear elasticity [2], and obtained an expression of the asymptotic expansion of the displacement field of a cylinder beam. He compared his results with the Nonlinear Strength of Materials [21], and specified how far its assumptions were valid. Since then, there have indeed

*Corresponding author: david@lmm.jussieu.fr; fax number: $(+33)$ 1.44.27.52.59. 
been numerous works using the asymptotic approach (see, among others, [11], [12], [13], [14], [15], [16], [17], [18]) but the Rigolot results have neither been used nor further developed. This lack of development is mainly due to the fact that A. Rigolot used a direct formulation of partial differential equations, contrary to other works which generally rely on variational formulations of the problems. A combination of variational theory and asymptotic expansion methods as in [19] could have been a powerful way to obtain all the terms of the asymptotic expansion.

The purpose of our work is to extend the Rigolot results, and determine explicitly any term of the asymptotic expansion. This is done through a new and original method used for the solving of recursive differential systems in nonlinear Mechanics: we show how it is possible to obtain the exact and explicit expression of any terms of the asymptotic expansion of the displacement field, and prove the asymptotic convergence of the series. The proposed method is a very general one and is not specific to our problem. Eventually, an application to plane bending of beams is presented.

\section{Position of the problem - Previous results}

Let us consider an elastic, linear, and isotropic cylinder beam, denoted by $\Xi$, of length $l$ and cross-sections $\Sigma_{0}$ and $\Sigma_{1}$, submitted to an external force $\mathbf{F}_{\mathbf{0}}$, of modulus $F_{0}$. Denoting by $d$ a specific quantity of $\Sigma_{0}$ (for example, its diameter), the slenderness of the cylinder is defined by $\epsilon$, where:

$$
\epsilon=\frac{d}{l}, \quad \epsilon<<1
$$

The slenderness of the beam plays the role of a gauge for the asymptotic expansion of the solution. The axes system $\mathbf{O} \mathbf{A}_{i},(i=1,2,3)$, is supposed principal, with central inertia for $\Sigma_{0} . \quad l$ is taken as unity of length and the study is based on the cylinder $\xi$ scaled from the cylinder $\Xi$ through the following change of variables (see Figure 1):
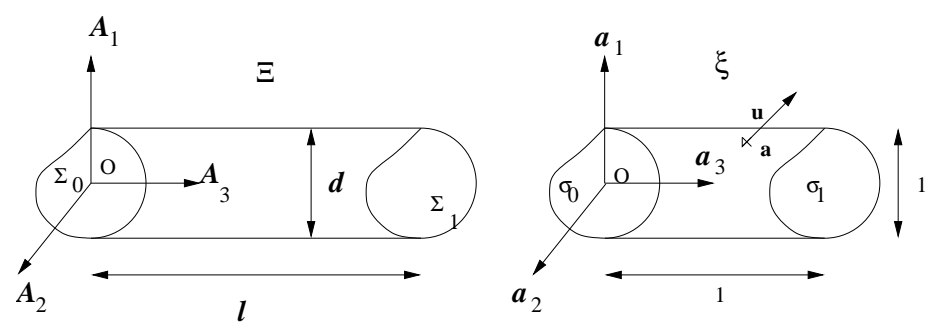

$\Xi$

Figure 1

$$
\begin{aligned}
a_{\alpha} & =\frac{A_{\alpha}}{d}, \\
a_{3} & =\frac{A_{3}}{l} .
\end{aligned}
$$


The Einsteinian summation convention applies to repeated indices, where Latin indices range from 1 to 3 whereas Greek indices range from 1 to 2. In [1], [2], Rigolot focused on the asymptotic approximation of the displacement field, modulo a given field $v$, for which he obtained the following expansion:

$$
\mathbf{u}=\sum_{n=0}^{\infty} \epsilon^{n} \mathbf{u}^{(n)}
$$

The components $u_{i}^{(n)}$ of the $n^{\text {th }}$ term of the above expansion are expressed in the coordinate system $\left(\mathbf{a}_{i}\right)$ related to the undeformed state of the beam. They are linked to the components of the field $\widetilde{\mathbf{u}}^{(n)}$, expressed in the coordinate system related to the deformed state of the beam through:

$$
u^{(n)}=A \widetilde{u}^{(n)}
$$

$A=\left[a_{i j}\right]_{1 \leq i \leq 3,1 \leq j \leq 3}$ is the transformation matrix from the initial state to the deformed one, expressed by means of the Euler angles:

$$
A=\left[\begin{array}{ccc}
\cos \varphi \cos \psi-\cos \theta \sin \varphi \sin \psi & -\cos \psi \sin \varphi-\cos \theta \cos \varphi \sin \psi & \sin \theta \sin \psi \\
\cos \theta \cos \psi \sin \varphi+\cos \varphi \sin \psi & \cos \theta \cos \varphi \cos \psi-\sin \varphi \sin \psi & -\cos \psi \sin \theta \\
\sin \theta \sin \varphi & \cos \varphi \sin \theta & \cos \theta
\end{array}\right]
$$

$\left(\mathbf{a}_{1}, \mathbf{a}_{2}, \mathbf{a}_{3}\right)$ and $\left(\mathbf{x}_{1}, \mathbf{x}_{2}, \mathbf{x}_{3}\right)$ being respectively the system coordinates of the initial and deformed state. Three rotations relate those two states, the angles of which are:

i. $\psi$, the precession angle;

ii. $\theta$, the nutation angle;

iii. $\phi$, the proper rotative angle.

In [1], [2], Rigolot showed that, for nonlinear elasticity of the second order, the $\widetilde{u}_{i}^{(n)}, n \geq 0$, are solutions of a differential recursive system of the form:

$$
\left\{\begin{array}{l}
\mathcal{L}_{\alpha \beta} \widetilde{u}_{\beta}^{(n+1)}=\Phi\left\{\mathbf{u}^{(1)}, \ldots, \mathbf{u}^{(n)}\right\} \\
\mathcal{B}_{\alpha \beta} \widetilde{u}_{\beta}^{(n+1)}=\Phi\left\{\mathbf{u}^{(1)}, \ldots, \mathbf{u}^{(n)}\right\}
\end{array}\right.
$$

( $\Phi$ being a generic notation denoting a function of $\left\{\mathbf{u}^{(1)}, \ldots, \mathbf{u}^{(n)}\right\}$ ) and that $\widetilde{u}_{3}^{(n+1)}$ satisfies the Neumann problem:

$$
\left\{\begin{array}{l}
\Delta \widetilde{u}_{3}^{(n+1)}=\Phi\left\{\mathbf{u}^{(1)}, \ldots, \mathbf{u}^{(n)}\right\} \\
\frac{d \widetilde{u}_{3}^{(n+1)}}{d n}=\Phi\left\{\mathbf{u}^{(1)} \ldots, \mathbf{u}^{(n)}\right\}
\end{array}\right.
$$


where $\mathcal{L}_{\alpha \beta}, \mathcal{B}_{\alpha \beta}$, and $\frac{d}{d n}$ are respectively the two dimensional elasticity operators, and border operators, defined by:

$$
\mathcal{L}_{\alpha \beta} u_{\beta}=\frac{2 \nu}{1-2 \nu} \frac{\partial}{\partial a_{\alpha}}\left(\frac{\partial u_{\gamma}}{\partial a_{\gamma}}\right)+\frac{\partial}{\partial a_{\beta}}\left(\frac{\partial u_{\alpha}}{\partial a_{\beta}}+\frac{\partial u_{\beta}}{\partial a_{\alpha}}\right) .
$$

in $\omega$, and:

$$
\left\{\begin{array}{l}
\mathcal{B}_{\alpha \beta} u_{\beta}=\frac{2 \nu}{1-2 \nu} \frac{\partial u_{\gamma}}{\partial a_{\gamma}} n_{\alpha}+\left(\frac{\partial u_{\alpha}}{\partial a_{\beta}}+\frac{\partial u_{\beta}}{\partial u_{\alpha}}\right) n_{\beta} \\
\frac{d u}{d n}=\frac{\partial u}{\partial a_{\alpha}} n_{\alpha}
\end{array}\right.
$$

on the lateral surface of $\omega$.

$\Delta$ is the two-dimensional laplacian, defined as:

$$
\Delta=\frac{\partial^{2}}{\partial a_{\alpha} \partial a_{\alpha}}
$$

In [3], it is shown that the Classical Strength of Materials for beams can be represented through the first two terms $\widetilde{\mathbf{u}}^{(0)}, \widetilde{\mathbf{u}}^{(1)}$, and that (10) enables us to express the components of $\widetilde{\mathbf{u}}^{(n+1)}, n \geq 0$, as functions of the components of the $\widetilde{\mathbf{u}}^{(n)}, n \geq 0$, modulo an unknown constant displacement:

$$
\begin{aligned}
& \widetilde{u}_{1}^{(n+1)}=-\nu\left(a_{i 3} \frac{\partial^{2} u_{i}^{(n)}}{\partial a_{3} \partial a_{1}} \frac{a_{1}^{2}-a_{2}^{2}}{2}+a_{i 3} \frac{\partial^{2} u_{i}^{(n)}}{\partial a_{3} \partial a_{2}} a_{1} a_{2}\right) \\
& \widetilde{u}_{2}^{(n+1)}=-\nu\left(a_{i 3} \frac{\partial^{2} u_{i}^{(1)}}{\partial a_{3} \partial a_{2}} \frac{a_{2}^{2}-a_{1}^{2}}{2}+a_{i 3} \frac{\partial^{2} u_{i}^{(n)}}{\partial a_{3} \partial a_{1}} a_{1} a_{2}\right) \\
& \widetilde{u}_{3}^{(n+1)}=-\frac{1}{2} \phi\left(a_{1}, a_{2}\right)^{T}\left[a_{1 i} \frac{\partial^{2}}{\partial a_{2} \partial a_{3}}-a_{i 2} \frac{\partial^{2}}{\partial a_{1} \partial a_{3}}\right] u_{i}^{(n)}
\end{aligned}
$$

$\phi^{T}$ denotes the torsional function.

\subsection{Calculation of the field $\mathbf{u}^{(n)}$}

We propose here, first, to use formal calculation to obtain the sequential terms of the asymptotic expansion of the solution.

Let us introduce the differential operating matrix $M^{\mathcal{D}}$, defined by:

$$
M^{\mathcal{D}}=\left[M^{\mathcal{D}}{ }_{i j}\right]_{1 \leq i \leq 3,1 \leq j \leq 3}
$$


where, for $j=1,2,3$ :

$$
\begin{aligned}
M^{\mathcal{D}}{ }_{1 j} & =-\nu a_{j 3}\left(\frac{a_{1}^{2}-a_{2}^{2}}{2} \frac{\partial^{2}}{\partial a_{1} \partial a_{3}}+a_{1} a_{2} \frac{\partial^{2}}{\partial a_{2} \partial a_{3}}\right) \\
M^{\mathcal{D}}{ }_{2 j} & =-\nu a_{j 3}\left(\frac{a_{2}^{2}-a_{1}^{2}}{2} \frac{\partial^{2}}{\partial a_{2} \partial a_{3}}+a_{1} a_{2} \frac{\partial^{2}}{\partial a_{1} \partial a_{3}}\right) \\
M^{\mathcal{D}}{ }_{3 j} & =-\frac{1}{2} \phi^{T}\left(a_{1}, a_{2}\right)\left(a_{j 1} \frac{\partial^{2}}{\partial a_{2} \partial a_{3}}-a_{j 2} \frac{\partial^{2}}{\partial a_{1} \partial a_{3}}\right)
\end{aligned}
$$

Relations (12), (13), (14), for $n \geq 1$, can then be written matricially as:

$$
\widetilde{\mathbf{u}}^{(n+1)}=M^{\mathcal{D}} \mathbf{u}^{(n)}
$$

where:

$$
\widetilde{\mathbf{u}}^{(2)}=\left[\begin{array}{c}
\widetilde{u}_{1}^{(2)} \\
\widetilde{u}_{2}^{(2)} \\
\widetilde{u}_{3}^{(2)}
\end{array}\right], \quad \mathbf{u}^{(1)}=\left[\begin{array}{c}
u_{1}^{(1)} \\
u_{2}^{(1)} \\
u_{3}^{(1)}
\end{array}\right]
$$

In the same way:

$$
\begin{aligned}
\widetilde{\mathbf{u}}^{(3)} & =M^{\mathcal{D}} \mathbf{u}^{(2)} \\
& =M^{\mathcal{D}}\left[A M^{\mathcal{D}}\right] \mathbf{u}^{(1)}
\end{aligned}
$$

Since, for any $n: \mathbf{u}^{(n)}=A \widetilde{\mathbf{u}}^{(n)}$, an immediate recurrence yields:

$$
\widetilde{\mathbf{u}}^{(n)}=M^{\mathcal{D}} \mathbf{u}^{(n-1)}
$$

In [3], it is shown that:

$$
\widetilde{\mathbf{u}}^{(1)}=M^{\mathcal{D}} \mathbf{u}^{(0)}
$$

Hence:

$$
\mathbf{u}^{(n+1)}=\left[A M^{\mathcal{D}}\right]^{n} \mathbf{u}^{(1)}
$$

which can be written as:

$$
\mathbf{u}^{(n+1)}=M^{n} \mathbf{u}^{(1)}
$$

where:

$$
M=A M^{\mathcal{D}}
$$

According to the recursive relation (26), the asymptotic expansion of the displacement field $\mathbf{u}$ can be written under the following form:

$$
\mathbf{u}=\sum_{n=0}^{\infty} \epsilon^{n} M^{n} \mathbf{u}^{(0)}
$$

The use of a symbolic formal tool (Mathematica [20]), enables us to calculate the formal product of two differential operating matrices, and therefore to obtain the $n^{\text {th }}$ power of the matrix $M$ : calculating any of the $\mathbf{u}^{(n)}$ is then possible.

In concrete terms, a finite truncation of the series (28) will serve the calculation of an approximation of the displacement field, as presented in section 4 . 


\section{Properties of the matrix $M$}

In the following, we show that the space of the solutions is a Fréchet space. For this purpose, properties of the space of differential operating matrices are first studied. Second, we prove the asymptotic convergence of the series (28).

\subsection{The space of matrices of differential operators}

Consider an open set $\omega$ of $\mathbb{R}^{n}$, and $\xi$ a compact set of $\omega$. Denote by $x=\left(x_{1}, \ldots, x_{n}\right)$ the current point of $\mathbb{R}^{n}$.

If $\alpha=\left(\alpha_{1}, \ldots, \alpha_{n}\right) \in \mathbb{N}^{n}$ is a multi-subscript with integer components, we will call length of $\alpha$ the integer $|\alpha|=\sum_{j=1}^{n} \alpha_{j}$.

Derivation with respect to the variable $x_{j},(1 \leq j \leq n)$, will be denoted by:

$$
D_{j}=\frac{\partial}{\partial x_{j}} \text {. }
$$

If $\alpha \in \mathbb{N}^{n}$ is a multi-index, we will note:

$$
D^{\alpha}=D_{1}^{\alpha_{1}} \cdots D_{n}^{\alpha_{n}}=\frac{\partial^{\alpha_{1}}}{\partial x_{1}{ }^{\alpha_{1}}} \cdots \frac{\partial^{\alpha_{n}}}{\partial x_{n}^{\alpha_{n}}}=\frac{\partial^{|\alpha|}}{\partial x_{1}^{\alpha_{1}} \cdots \partial x_{n}^{\alpha_{n}}}
$$

Consider, in $\mathbb{R}^{n}$, a square $N \times N$ linear differential operating matrix, whose coefficients are $C^{\infty}$.

Denote this matrix $M$. Set:

$$
M=\left[a_{i j}(x, D)\right]_{1 \leq i, j \leq N}
$$

The order of the operator $a_{i j}(x, D)$ will be denoted by $m_{i j}$ :

$$
m_{i j}=\operatorname{order}\left(a_{i j}(x, D)\right)
$$

If the operator is identically zero, its order will be $-\infty$.

If $m_{i j}$ is finite $(<\infty)$, we have then:

$$
a_{i j}(x, D)=\sum_{|\alpha| \leq m_{i j}} a_{i j, \alpha}(x) D^{\alpha}
$$

where the functions $a_{i j, \alpha}: \omega \longrightarrow \mathbb{R}$ are $C^{\infty}$.

To the matrix $M=\left[a_{i j}(x, D)\right]_{1 \leq i, j \leq N}$, we relate the square $N \times N$ matrix, whose coefficients are in $\mathbb{N} \cup\{-\infty\}$ :

$$
\left[m_{i j}\right]_{1 \leq i, j \leq N}
$$


which will be called matrix of the related orders of the matrix $M, M=\left[a_{i j}(x, D)\right]_{1 \leq i, j \leq N}$.

To each operator $a_{i j}(x, D)$, we relate a polynomial with $n$ unknowns $\psi_{i}, i=1, \ldots, n$ in the following way: if $m_{i j}=-\infty$, the polynomial $a_{i j}(x, \psi)$ related to the operator $a_{i j}(x, D)$ is equal to zero, or else it is the polynomial of degree $m_{i j}$ defined by:

$$
a_{i j}(x, \psi)=\sum_{|\alpha| \leq m_{i j}} a_{i j, \alpha}(x) \psi^{\alpha}
$$

where $\psi^{\alpha}=\psi_{1}^{\alpha_{1}} \ldots \psi_{n}^{\alpha_{n}}$.

We thus define a square $N \times N$ polynomial matrix:

$$
\left[a_{i j}(x, \psi)\right]_{1 \leq i, j \leq N}
$$

Denote by $\mathcal{P}$ the set of permutations of $[1, N]$. For any permutation $\pi \in \mathcal{P}$, we set:

$$
m(\pi)=\sum_{i=1}^{N} m_{i, \pi(i)}
$$

The determinant of the matrix defined in (34) is a polynomial of degree less or equal to:

$$
m_{\mathcal{P}}=\sup _{\pi \in \mathcal{P}} m(\pi)
$$

$m_{\mathcal{P}}$ will be called total order of the system (29). $m_{\mathcal{P}}$ can be equal to $-\infty$, which is, for instance, the case if there exists a line (or a column) of the matrix (29) identically zero. We will always assume that $m_{\mathcal{P}}$ is positive.

Let us denote by $g(x, \psi)$ the homogeneous part of degree $m_{\mathcal{P}}$ of the polynomial $\operatorname{det}\left[a_{i j}(x, \psi)\right]$; this polynomial is called the characteristic polynomial of $(29)$. We will take for granted that for any $x$ in $\omega, g$ is not identically zero.

Denote by $\mathcal{M}^{N}$ the space of square $N \times N$ linear differential operating matrices, operating on the function space $U$ of the $C^{\infty}$ bounded functions on $\Omega$ (we recall, here, that the displacement solution $\mathbf{u}$ is, by construction, $C^{\infty}$, and therefore bounded on $\Omega$ ).

Let $M$ be in $\mathcal{M}^{N}$, thus its components are of the form:

$$
a_{i j}(x, D)=\sum_{|\alpha| \leq m_{i j}} a_{i j, \alpha}(x) D^{\alpha} \quad 1 \leq i, j \leq N
$$

$\mathcal{M}^{N}$ is a $\mathbb{C}$-vectorial space of finite dimension:

if, for $1 \leq i, j \leq N, E_{i j}$ is the matrix whose coefficients of which are the identically zero operators, except the one of subscript $(i, j)$, which is the identity operator, denoted by 1 .

The canonic basis of $\mathcal{M}^{N}$ is given by:

$$
\left\{\frac{\partial^{\alpha_{i j}^{1}} \ldots \partial^{\alpha_{i j}^{n}}}{\partial_{x_{1}}^{\alpha_{i j}^{1}} \ldots \partial_{x_{n}}^{\alpha_{i j}^{n}}} E_{i j}\right\}, \quad 1 \leq i, j \leq N
$$


where, for any $(i, j)$, the $\alpha_{i j}^{k}$ are integers, corresponding to the differentiation order with respect to the variable $x_{k}$.

Note: $m_{0}=\max _{1 \leq i \leq N, 1 \leq j \leq N} m_{i j}$; we presently study the case where, for any $(i, j), m_{i j}$ is finite.

Let us review what a combination with repetitions is: $E$ being a set of $n$ elements, a combination with repetition of $p$ elements of $E$ is any set of $p$ elements $\left\{x_{1}, \cdots, x_{p}\right\}$ not necessarily distinct. The number of combinations with repetitions of $p$ elements among $n$ is $C_{n+p-1}^{p}$.

Thus, for any differentiation order $p$, the number of combinations of partial differentiations with respect to the variables $x_{1}, \cdots, x_{n}$, that is the number of combinations with repetition of $p$ elements taken in the set of $n$ elements $\left\{x_{1}, \cdots, x_{n}\right\}$ is $C_{n+p-1}^{p}$. Or, $m_{0}$ being defined by: $m_{0}=\max _{1 \leq i \leq N, 1 \leq j \leq N} m_{i j}$, for any subscript $(i, j)$, $1 \leq i, j \leq N$, there are $\sum_{p=0}^{m_{0}} C_{n+p-1}^{p}$ choices available. Eventually:

$$
\operatorname{dim}\left(\mathcal{M}^{N}\right)=\left[\sum_{p=0}^{m_{0}} C_{N+p-1}^{p}\right]^{N^{2}}
$$

It is important to note that the $\mathbf{u}^{(n)}$ terms we presently work on are, by construction, $C^{\infty}$, with a compact support $\xi$ : so are all their derivatives. Of course, the $(n+1)^{t h}$ term is related to the $n^{\text {th }}$ one by means of a differential system. Since all those terms are defined on a compact set, they are bounded. It is then interesting to note that all the $\mathbf{u}^{(n)}$ terms still belong to this peculiar space.

Therefore, any of the $M \mathbf{u}^{(n)}$ term is still in $U$, which means that applying a differential operating matrix of $\mathcal{M}^{N}$ on any of the $\mathbf{u}^{(n)}$ terms results in obtaining a term in $U$.

We then define on $\mathcal{M}^{N}$ an associative and distributive multiplication, denoted by ".", i.e. for $M, N$ and $P$ in $\mathcal{M}^{N}$ :

$$
\begin{aligned}
M \cdot(N \cdot P) & =(M \cdot N) \cdot P, \\
(M+N) \cdot P & =M \cdot N+N \cdot P, \\
M \cdot(N+P) & =M \cdot N+M \cdot P
\end{aligned}
$$

Multiplication by a scalar satisfies:

$$
\lambda(M N)=M(\lambda N)=(\lambda M) N, \lambda \in \mathbb{C} .
$$

We provide $\mathcal{M}^{N}$ with the norm $\|||$.$\| , defined by:$

$$
\text { for } M \in \mathcal{M}^{N} \quad\|M\|=\sum_{i, j=1}^{N}\left\|a_{i j}(x, D)\right\|_{\mathcal{A}}
$$

where \|\|$_{\mathcal{A}}$ is an algebra norm on the algebra $\mathcal{A}$ of linear differential operators, defined as follows:

let us recall, first, that an element $a(x, D)$ of $\mathcal{A}$ can be written as:

$$
a(x, D)=\sum_{k \in I} a_{k, 1}(x) D^{\alpha^{k, 1}} \ldots a_{k, p_{k}}(x) D^{\alpha^{k, p_{k}}}
$$


where $I$ is a finite set, $\alpha^{k, l} \in \mathbb{N}^{n}, a_{k, l},\left(k \in I, 1 \leq l \leq p_{k}\right), C^{\infty}$ functions. For any $(i, j), 1 \leq i, j \leq N$, we have :

$$
\begin{aligned}
a_{i j}(x, D): \mathcal{X} & \longrightarrow \mathcal{X} \\
u_{j} & \longrightarrow a_{i j}(x, D) u_{j}
\end{aligned}
$$

where $\mathcal{X}$ is the function space to which belong the components $u_{j}, j \in[1, N]$ of the displacement field $\mathbf{u}$.

$\mathcal{A}$ is then provided with the norm:

$$
\left\|a_{i j}(x, D)\right\|_{\mathcal{A}}=\sup _{\left\|u_{j}\right\|_{\mathcal{X}} \leq 1}\left\|a_{i j}(x, D) u_{j}\right\|_{\mathcal{X}}
$$

Moreover, if $\left\|a_{i j}(x, D)\right\|_{\mathcal{A}}<\infty$ (i.e. the operator $a_{i j}(x, D)$ is continuous) and $\mathcal{X}$ is a Banach space, then $\mathcal{A}$ is a Banach algebra.

Norms being equivalent in the space $\mathcal{M}^{N}$, we can also take:

$$
\|M\|=\sup _{\|\mathbf{u}\|_{\mathcal{X} \leq 1}}\|M u\|_{\mathcal{X}}
$$

In both cases, ||$|\cdot|||$ is an algebra norm, i.e.:

$$
\text { for } \quad M, N \in \mathcal{M}^{N}: \quad\|M . N|\|\leq\|| M|\|\cdot|\|N \mid\|
$$

$\mathcal{M}^{N}$ is then a normed algebra. Moreover, $\mathcal{M}^{N}$ being a vector normed space of finite dimension, $\mathcal{M}^{N}$ is a Banach space.

Thus, $\mathcal{M}^{N}$ is a non-commuting unitary (i.e. with an identity element) Banach algebra.

The study of the norm of an element of $\mathcal{M}^{N}$ depends on the components $u_{j}, \quad 1 \leq$ $j \leq N$, of the field $\mathbf{u}$.

For the space $\mathcal{X}$, we will limit our study to the general case of a Sobolev space $H_{m}(\omega)$, in so far as the Rigolot results [1], [2], ensure that the solution $\mathbf{u}$ belongs to $H_{m}(\omega)$, and has a compact support $\xi \subset \omega$.

Denote by $\mathcal{E}_{m}(\omega)$ the function space of the $\mathbf{u} \in C^{\infty}(\omega)$ such that:

$$
\left|D^{\alpha} \mathbf{u}\right| \in L_{2}(\omega), \quad \forall 0 \leq|\alpha| \leq m \in \mathbb{N} .
$$

the Sobolev space $H_{m}(\omega)$ is the completion of $\mathcal{E}_{m}(\omega)$, with respect to the norm:

$$
\|\mathbf{u}\|_{H_{m}}=\sum_{|\alpha|=0}^{m}\left\|D^{\alpha} \mathbf{u}\right\|_{L_{2}}
$$

where

$$
\left\|D^{\alpha} \mathbf{u}\right\|_{L_{2}}=\left[\int_{\Omega}\left|D^{\alpha} \mathbf{u}\right|^{2}\right]^{\frac{1}{2}}
$$

Hence,

$$
H_{m}(\omega)=\left\{\mathbf{u} \in L_{2}(\Omega), D^{\alpha} \mathbf{u} \in L_{2}(\Omega), \quad|\alpha| \leq m\right\}
$$


The components $u_{j}, 1 \leq j \leq N$, of the displacement field $\mathbf{u}$, belong to $H_{m}(\omega)$. Its norm is given by:

$$
\|\mathbf{u}\|_{H_{m}}=\left[\sum_{j=1}^{N}\left\|u_{j}\right\|_{H_{m}}^{2}\right]^{\frac{1}{2}}
$$

Let $M \in \mathcal{M}^{N}$. Then:

$$
\begin{aligned}
M & =\left[a_{i j}(x, D)\right]_{1 \leq i, j \leq N} \\
& =\left[\sum_{|\alpha| \leq m_{i j}} a_{i j, \alpha}(x) D^{\alpha}\right]_{1 \leq i, j \leq N}
\end{aligned}
$$

The coefficients $a_{i j, \alpha}(x)$ are real and continuous.

$M$ generates a linear operator in the vectorial function space $\overline{L_{2}(\Omega)}$ of the square modulus integrable functions.

Each coefficient of the matrix is a linear differential operator, defined by:

$$
\begin{aligned}
\sum_{|\alpha| \leq m_{i j}} a_{i j, \alpha}(x) D^{\alpha}: H_{m}(\Omega) & \longrightarrow H_{m}(\Omega) \\
u_{j} & \mapsto \sum_{|\alpha| \leq m_{i j}} a_{i j, \alpha}(x) D^{\alpha} u_{j}
\end{aligned}
$$

where $m=\max _{i, j} m_{i j}$.

The space $\mathcal{M}^{N}$ is provided with the norm (40):

$$
\begin{aligned}
\|M\| \| & =\sup _{\|\mathbf{u}\|_{H_{m}} \leq 1}\|M \cdot \mathbf{u}\|_{H_{m}} \\
& =\sup _{\|\mathbf{u}\|_{H_{m}} \leq 1}\left[\sum_{i=1}^{N}\left\|(M . \mathbf{u})_{i}\right\|_{H_{m}}^{2}\right]^{\frac{1}{2}}
\end{aligned}
$$

where $(M . \mathbf{u})_{i}$ is the $i^{\text {th }}$ component of the vector $M . \mathbf{u}$ and:

$$
\begin{aligned}
(M . \mathbf{u})_{i} & =\sum_{j=1}^{N} a_{i j}(x, D) u_{j} \\
& =v_{i}
\end{aligned}
$$

Hence:

$$
\begin{aligned}
\|\| M\|\| & =\sup _{\|u\|_{H_{m}} \leq 1}\left[\sum_{i=1}^{N}\left\|\sum_{j=1}^{3} a_{i j}(x, D) u_{j}\right\|_{H_{m}}^{2}\right]^{\frac{1}{2}} \\
& =\sup _{\|u\|_{H_{m}} \leq 1}\left[\sum_{i=1}^{N}\left\|v_{i}\right\|_{H_{m}}^{2}\right]^{\frac{1}{2}} \\
& =\sup _{\|u\|_{H_{m}} \leq 1}\left[\sum_{i=1}^{N}\left(\sum_{|\beta| \leq m}\left\|D^{\beta} v_{i}\right\|_{L_{2}}\right)^{2}\right]^{\frac{1}{2}}
\end{aligned}
$$


For any $i \in\{1, \ldots, N\}, v_{i}$ belongs to $H_{m}(\Omega)$, and has a compact support $\xi \subset \omega$; thus $D^{\beta} v_{i} \in L_{2}(\Omega)$ for any integer $\beta$, with $0 \leq|\beta| \leq m$; if $D^{\beta} v_{i} \in L_{2}(\Omega)$, and has a compact support $\xi \subset \omega$, then $\left\|D^{\beta} v_{i}\right\|_{L_{2}}<\infty$.

Hence, there exists a constant $C \geq 0$ such that:

$$
\begin{aligned}
\|M\| \| & =\sup _{\|u\|_{H_{m}} \leq 1}\|M \cdot \mathbf{u}\|_{H_{m}} \\
& \leq C
\end{aligned}
$$

\subsection{Asymptotic convergence of the series (28)}

The asymptotic expansion of the displacement field $\mathbf{u}$ can be written as:

$$
u=\sum_{n=0}^{\infty} \epsilon^{n} M^{n} u^{(0)}
$$

The validity of the asymptotic expansion will be assessed through the asymptotic convergence of the series $\sum_{n=0}^{\infty} \epsilon^{n} M^{n}$.

Let us first demonstrate the following lemma:

Lemma 3.2.1 Consider a $C^{\infty}$ function $f$, with a closed and bounded support $J$. Denote by $x_{0}$ a point of $J$, and $\epsilon$ a strictly positive real number, very small compared to 1.

Then: the series $\left(\sum \epsilon^{n} f^{(n)}\left(x_{0}\right)\right)$ is asymptotically convergent.

Proof 3.2.1 Let $x$ be a point of $J$, very close from $x_{0}$.

We will take, in the following:

$$
\epsilon=\eta\left|x-x_{0}\right|
$$

with $\eta \ll 1$.

Let $n$ be a strictly positive natural integer. The Taylor-Young formulae, at the first order, applied to $f^{(n-1)}$ at the point $x_{0}$, can be written as:

$$
f^{(n-1)}(x)=f^{(n-1)}\left(x_{0}\right)+\left(x-x_{0}\right) f^{(n)}\left(x_{0}\right)+o(1)
$$

Therefore:

$$
\left|\epsilon^{n} f^{(n)}\left(x_{0}\right)\right|=\epsilon^{n-1} \eta\left|x-x_{0}\right|\left|f^{(n)}\left(x_{0}\right)\right|=\epsilon^{n-1} \eta\left|f^{(n-1)}(x)-f^{(n-1)}\left(x_{0}\right)+o(1)\right|
$$

$f$ being $C^{\infty}$ on $J$, all the derivatives $f^{(k)}(k \geq 1)$ are continuous at the point $x_{0}$ : for any strictly positive integer $n$, there exists an interval $] x_{0}-\delta_{n}, x_{0}+\delta_{n}\left[\right.$ of center $x_{0}$, where:

$$
\left|f^{(n-1)}(x)-f^{(n-1)}\left(x_{0}\right)+o(1)\right| \leq 1
$$

We have then:

$$
\left|\epsilon^{n} f^{(n)}\left(x_{0}\right)\right| \leq \epsilon^{n-1} \eta
$$


Let now $\alpha$ be a strictly positive real number, and $N_{0}$ a strictly positive integer. Let us introduce the sequence defined by:

$$
S_{N_{0}}=\sum_{n=1}^{N_{0}} \epsilon^{n} f^{(n)}\left(x_{0}\right)
$$

Using:

$$
I_{N_{0}}=\overbrace{n=1}^{N_{0}}] x_{0}-\delta_{n}, x_{0}+\delta_{n}[
$$

we obtain:

$$
\left|\sum_{n=1}^{N_{0}} \epsilon^{n} f^{(n)}\left(x_{0}\right)\right| \leq \sum_{n=1}^{N_{0}} \eta \epsilon^{n-1}=\eta\left\{\frac{1-\epsilon^{N_{0}-1}}{1-\epsilon}\right\} \leq \frac{\eta}{1-\epsilon}
$$

or:

$$
\left|S_{N_{0}}\right| \leq \frac{\eta}{1-\epsilon}
$$

This lemma can easily be generalized to the differential operating matrix $M$ : we use for this purpose its decomposition on the basis of $\mathcal{M}^{N}$. Each component of $M$ is a linear combination of differential operators, to which the lemma can be applied. More specifically, results of subsection 3. 1. enable us to assert that, for the $u$ with a compact support $\xi \subset \omega$ of $H_{m}(\Omega)$, for $N_{0}$ in $\mathbb{N}$, there exists a strictly positive real number $\epsilon$ which satisfies:

$$
\text { for } 1 \leq n \leq N_{0}: \quad\left\|\epsilon M^{n} u^{(0)}\right\| \leq 1
$$

Hence:

$$
\left\|\sum_{n=1}^{N_{0}} \epsilon^{n} M^{n} u^{(0)}\right\| \leq \sum_{n=1}^{N_{0}} \epsilon^{n-1}\left\|\epsilon M^{n} u^{(0)}\right\| \leq \frac{1}{1-\epsilon}
$$

The series $\sum_{n=0}^{\infty} \epsilon^{n} M^{n} u^{(0)}$ is then asymptotically convergent.

\subsection{A property of the solution}

In the above subsection, we have concentrated our study on differential operating matrices spaces. This was the necessary step to a full understanding of the behavior of the components $\mathbf{u}^{(n)}$ of the asymptotic expansion of the solution.

Let us denote by $\mathcal{U}$ the space $\operatorname{Vect}\left\{\mathbf{u}^{(n)}, n \in \mathbb{N}\right\}$.

We provide $\mathcal{U}$ with the semi-norm family:

$$
\|\mathbf{v}\|=\sup \|M \cdot \mathbf{v}\|_{H_{m}, m \in \mathbb{N}} \mathbf{v} \in \mathcal{U}
$$

Any $\mathbf{v}$ in $\mathcal{U}$ has a compact support $\xi . \mathcal{U} \subset C_{\xi}^{\infty}$ is a subset of the Frechet space $C_{\xi}^{\infty}$ for the semi-norm family (67). This property is all the more useful as a Cauchy sequence of this space will converge, and thus accounts for a finite truncation of the field (28). This property is used in section 4., the difference between two successive terms $\epsilon^{n} u^{(n)}$ and $\epsilon^{n+1} u^{(n+1)}$ being small enough up from a specific rank $n$. 


\section{Numerical Application}

The asymptotic solution field, in the case of plane bending, for a free-embedded cantilever, subject to a constant moment of flexion, is presented in the following (see Figure 2).

We will denote by $\nu$ Poisson's ratio.

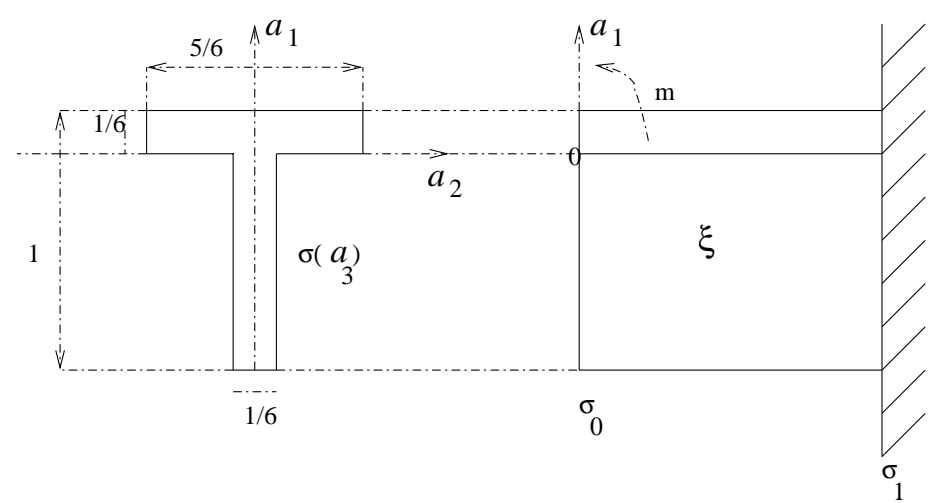

Figure 2: The free-embedded cantilever subject to a constant moment of flexion

The rotation matrix $A$ depends only on the angular parameter $\theta$, which itself solely depends on $a_{3}$, the two remaining Euler angles being constant. $A$ is here of the following form:

$$
A=\left[\begin{array}{ccc}
\cos \left[\frac{m}{I}\left(a_{3}-1\right)\right] & 0 & \sin \left[\frac{m}{I}\left(a_{3}-1\right)\right] \\
0 & 1 & 0 \\
-\sin \left[\frac{m}{I}\left(a_{3}-1\right)\right] & 0 & \cos \left[\frac{m}{I}\left(a_{3}-1\right)\right]
\end{array}\right]
$$

where $m$ is the modulus of the moment of flexion.

Under those assumptions, the differential operating matrix, denoted by $M$, which relates $\mathbf{u}^{(n+1)}$ and $\mathbf{u}^{(n)}$, satisfies:

$$
M=\left[\begin{array}{ccccc}
-\nu \sin \frac{m\left(a_{3}-1\right)}{I} & \frac{1}{2} \frac{\left(a_{1}^{2}-a_{2}^{2}\right) \partial^{2}}{\partial a_{1} \partial a_{3}}+\frac{a_{1} a_{2} \partial^{2}}{\partial a_{2} \partial a_{3}} & 0 & -\nu \cos \frac{m\left(a_{3}-1\right)}{I} & \frac{1}{2} \frac{\left(a_{1}^{2}-a_{2}^{2}\right) \partial^{2}}{\partial a_{1} \partial a_{3}}+\frac{a_{1} a_{2} \partial^{2}}{\partial a_{2} \partial a_{3}} \\
\nu \sin \frac{m\left(a_{3}-1\right)}{I} & \frac{1}{2} \frac{\left(a_{1}^{2}-a_{2}^{2}\right) \partial^{2}}{\partial a_{2} \partial a_{3}}+\frac{a_{1} a_{2} \partial^{2}}{\partial a_{1} \partial a_{3}} & 0 & -\nu \cos \frac{m\left(a_{3}-1\right)}{I} & \frac{1}{2} \frac{\left(a_{1}^{2}-a_{2}^{2}\right) \partial^{2}}{\partial a_{2} \partial a_{3}}+\frac{a_{1} a_{2} \partial^{2}}{\partial a_{1} \partial a_{3}}
\end{array}\right]
$$

Denote by $I$ the inertia moment of the beam. The field $u^{(0)}$ is solution of the differential system (see [3]):

$$
\left\{\begin{aligned}
\frac{\partial^{2} u_{2}(0)}{\partial a_{3}{ }^{2}}\left(0,0, a_{3}\right) & =0 \\
\frac{\partial}{\partial a_{3}}\left[\cos \frac{m\left(a_{3}-1\right)}{I} \frac{\partial u_{1}(0)}{\partial a_{3}}\left(0,0, a_{3}\right)-\sin \frac{m\left(a_{3}-1\right)}{I} \frac{\partial u_{3}(0)}{\partial a_{3}}\left(0,0, a_{3}\right)\right]\left(0,0, a_{3}\right) & =C \\
\sin \frac{m\left(a_{3}-1\right)}{I} \frac{\partial u_{1}(0)}{\partial a_{3}}\left(0,0, a_{3}\right)+\cos \frac{m\left(a_{3}-1\right)}{I} \frac{\partial u_{3}(0)}{\partial a_{3}}\left(0,0, a_{3}\right) & =0 \\
\widetilde{u}_{i}(0)(0,0,1)=\frac{\partial u_{\alpha}(0)}{\partial a_{3}}(0,0,1) & =0
\end{aligned}\right.
$$

where:

$$
C=\frac{3}{2 \varepsilon I} 0.0199\{(1-\nu)-0.0005 \nu\}
$$


The resolution of (70) yields:

$$
\left\{\begin{array}{l}
u_{1}^{(0)}\left(0,0, a_{3}\right)=\frac{C I^{2}}{m^{2}}\left\{\cos \left[\frac{m\left(a_{3}-1\right)}{I}\right]-1\right\}+\frac{C I}{m}\left(a_{3}-1\right) \sin \left[\frac{m\left(a_{3}-1\right)}{I}\right] \\
u_{3}^{(0)}\left(0,0, a_{3}\right)=-\frac{C I^{2}}{m^{2}} \sin \left[\frac{m\left(a_{3}-1\right)}{I}\right]+\frac{C I}{m}\left(a_{3}-1\right) \cos \left[\frac{m\left(a_{3}-1\right)}{I}\right]
\end{array}\right.
$$

The hypothesis of small deformations leads to:

$$
u_{i}^{(0)}\left(a_{1}, a_{2}, a_{3}\right)=\frac{\partial u_{i}^{(0)}}{\partial a_{1}}\left(0,0, a_{3}\right) a_{1}+\frac{\partial u_{i}^{(1)}}{\partial a_{2}}\left(0,0, a_{3}\right) a_{2}+u_{i}^{(0)}\left(0,0, a_{3}\right)
$$

Due to (see [3]):

$$
\begin{aligned}
& \frac{\partial u_{1}^{(0)}}{\partial a_{1}}\left(0,0, a_{3}\right)=a_{11}-1=\cos \left[\frac{m\left(a_{3}-1\right)}{I}\right]-1 \\
& \frac{\partial u_{1}^{(0)}}{\partial a_{2}}\left(0,0, a_{3}\right)=a_{12}=0 \\
& \frac{\partial u_{3}^{(0)}}{\partial a_{1}}\left(0,0, a_{3}\right)=a_{31}=-\sin \left[\frac{m\left(a_{3}-1\right)}{I}\right] \\
& \frac{\partial u_{3}^{(0)}}{\partial a_{2}}\left(0,0, a_{3}\right)=a_{32}=0
\end{aligned}
$$

we then have:

$$
\left\{\begin{array}{l}
u_{1}^{(0)}\left(a_{1}, a_{2}, a_{3}\right)=\left(\frac{C I^{2}}{m^{2}}+a_{1}\right)\left\{\cos \left[\frac{m\left(a_{3}-1\right)}{I}\right]-1\right\}+\frac{C I}{m}\left(a_{3}-1\right) \sin \left[\frac{m\left(a_{3}-1\right)}{I}\right] \\
u_{2}^{(0)}\left(a_{1}, a_{2}, a_{3}\right)=0 \\
u_{3}^{(0)}\left(a_{1}, a_{2}, a_{3}\right)=-\left(\frac{C I^{2}}{m^{2}}+a_{1}\right) \sin \left[\frac{m\left(a_{3}-1\right)}{I}\right]+\frac{C I}{m}\left(a_{3}-1\right) \cos \left[\frac{m\left(a_{3}-1\right)}{I}\right]
\end{array}\right.
$$

Symbolic calculations yield:

$$
\begin{gathered}
\left\{\begin{array}{l}
u_{1}{ }^{(1)}=\frac{0.5 m\left(a_{1}^{2}-a_{2}^{2}\right) \nu \cos \left[\frac{m\left(a_{3}-1\right)}{I}\right]}{I} \\
u_{2}{ }^{(1)}=\frac{m a_{1} a_{2} \nu}{I} \\
u_{3}{ }^{(1)}=-\frac{0.5 m\left(a_{1}^{2}-a_{2}^{2}\right) \nu \sin \left[\frac{m\left(a_{3}-1\right)}{I}\right]}{I}
\end{array}\right. \\
\left\{\begin{array}{l}
u_{1}{ }^{(2)}=\frac{0.5 m^{2} a_{1}\left(a_{1}^{2}-3 a_{2}^{2}\right) \nu^{2} \cos \left[\frac{m\left(a_{3}-1\right)}{I}\right]}{I^{2}} \\
u_{2}{ }^{(2)}=\frac{\left.0.5 m^{2} a_{2}\left(3 a_{1}^{2}\right)-a_{2}^{2}\right) \nu^{2}}{I^{2}} \\
u_{3}{ }^{(2)}=-\frac{0.5 m^{2} a_{1}\left(a_{1}^{2}-3 a_{2}^{2}\right) \nu^{2} \sin \left[\frac{m\left(a_{3}-1\right)}{I}\right]}{I^{2}}
\end{array}\right.
\end{gathered}
$$




$$
\left\{\begin{array}{l}
u_{1}{ }^{(3)}=\frac{0.75 m^{3}\left(a_{1}^{4}-6 a_{1}^{2} a_{1}^{2}+a_{2}^{4}\right) \nu^{3} \cos \left[\frac{m\left(a_{3}-1\right)}{I}\right]}{I_{2}{ }^{3} a_{1} a_{2}\left(a_{1}^{2}-a_{2}^{2}\right) \nu^{3}} \\
u_{2}{ }^{3} \\
u_{3}{ }^{(3)}=-\frac{0.75 m^{3}\left(a_{1}^{4}-6 a_{1}^{2} a_{1}^{2}+a_{2}^{4}\right) \nu^{3} \sin \left[\frac{m\left(a_{3}-1\right)}{I}\right]}{I^{3}}
\end{array}\right.
$$

Higher order terms are calculated in the same way. Up from rank 3, the difference

$$
\sup _{\left(a_{1}, a_{2}, a_{3}\right) \in \Omega}\left|\epsilon^{n+1} u^{(n+1)}\left(a_{1}, a_{2}, a_{3}\right)-\epsilon^{n} u^{(n)}\left(a_{1}, a_{2}, a_{3}\right)\right|
$$

begins to become very small, which can account for the validity of a finite truncation of the field

$$
u=\sum_{n=0}^{\infty} \epsilon^{n} M^{n} u^{(0)}
$$

Figure 3 displays the third component, calculated respectively at the second and third order, of the field $u$, for given values of $a_{1}$ and $a_{2}$, as a function of the normalized thickness variable $\overline{a_{3}}$, and the small parameter $\varepsilon$. The two plots coincide, which accounts for the accuracy of the truncature at the second order.

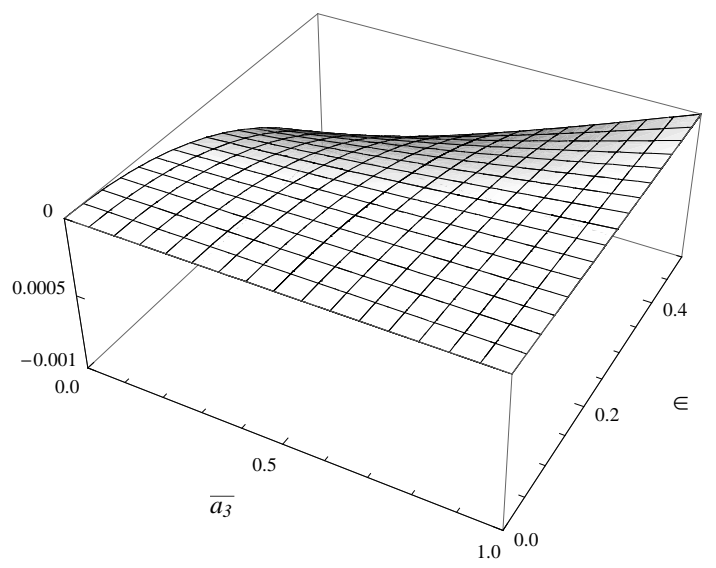

Figure 3: The third component of the field $u$, for $a_{1}=a_{2}=0.5$, at the second and third orders, as a function of the normalized thickness variable $\overline{a_{3}}$, and the small parameter $\varepsilon$

\section{Conclusion}

The approximate displacement field, solution of Saint-Venant's problem, for the elasticity of the second order, is obtained as the sum of a series, which is geometric as regards the inverse of the slenderness of the beam. This series is shown to be asymptotically convergent. The terms of the series can be calculated recursively using a symbolic calculus tool. The accuracy of the proposed method is shown in the case of plane bending, for a free-embedded cantilever beam. For this specific case, the influence of the components of the asymptotic expansion come clearly into light: a truncation at the second order appears as sufficiently accurate. 


\section{Acknowledgment}

The authors would like to thank Prof. E. Sanchez-Palencia for the interest he took in their work, and for his judicious remarks and advices.

\section{References}

[1] Rigolot, A., Sur une théorie asymptotique des poutres, Journal de Mécanique, 11(44): 673-703, 1972.

[2] Rigolot, A., Sur une théorie asymptotique des poutres droites, Thèse de Doctorat d'Etat, Université Paris 6, France, 1976.

[3] Rigolot, A., Déplacements finis et petites déformations des poutres droites : Analyse asymptotique de la solution à grande distance des bases, Journal de la Mécanique appliquée, 1(2): 175-206, 1977.

[4] Rigolot, A., Approximation asymptotique des vibrations de flexion des poutres droites élastiques, Journal de Mécanique, 16(4): 493-529, 1977.

[5] Hay, G. E., The finite displacements of thin rods, Trans. of the Am. Math. Soc., 51(2): 65-102, 1942.

[6] Muller, P., Etude des déplacements finis d'une tige hyperélastique incompressible, Thèse de Doctorat de troisième cycle, Faculté des Sciences de Paris, France, 1970 .

[7] Antman, S., The Theory of Rods, Handbuch des Physik. Springer, Berlin, VI(a/2): 641-700, 1972.

[8] Plumat, Y., Contribution à l'étude asymptotique des poutres circulaires, Thèse de Doctorat de troisième cycle, Faculté des Sciences de Paris, France, 1976.

[9] Germain, P., Cours de mécanique des milieux continus, Masson, Paris, 1973.

[10] Lions, J.-L. and Magenes, E., Problèmes aux limites non homogènes et applications. Dunod, 1970.

[11] Ciarlet, P. G., Destuynder, P. A justification of nonlinear model in plate theory, Computer methods in Applied Mechanics and engineering, 17/18: 227-258, 1979 .

[12] Ciarlet, P. G., Destuynder, P., A justification of the two-dimensinal linear plate model. J. Mécanique, 17/18: 227-258, 1979.

[13] Cimetière, A., Geymonat, G., Le Dret, H., Raoult, A., Tutek, Z., A derivation of a nonlinear rod model from three-dimensional elasticity. C. R. Acad. Sc. Paris, 302(I)(19): 697-700, 1986. 
[14] Cimetière, A., Geymonat, G., Le Dret, H., Raoult, A., Tutek, Z., Asymptotic theory and analysis for displacements and stress distribution in nonlinear elastic straight slender rods. J. Elasticity, 19: 111-161, 1988.

[15] Alvarez-Vazquez, L.-J., Samartin, A. and Viano, J.-M., Mathematical model for elastic beams with longitudinally variable depth. Asymptotic Analysis, 27: 75-93, 2001.

[16] Sanchez-Hubert J., Sanchez-Palencia E., Couplage flexion-torsion-traction dans les poutres anisotropes à section hétérogène. C. R. Acad. Sci. Paris, 312(2): 337-344,1991.

[17] Jamal R., Sanchez-Palencia E., Théorie asymptotique des tiges courbes anisotropes. C. R. Acad. Sci. Paris, 322(1): 1099-1106, 1996.

[18] Sanchez-Hubert J. Sanchez-Palencia E., Statics of curved rods on account of torsion and flexion. Eur. J. Mech. A/Solids, 18: 365-390, 1999.

[19] Trabucho, L., Viaño, J. M., Mathematical modelling of rods, Handbook of numerical analysis, IV, 487-974, North-Holland, Amsterdam, 1996.

[20] Wolfram, S., The Mathematica Book, Wolfram Media, Fourth Edition, 1999.

[21] Courbon, J., Résistance des Matériaux, Dunod, Paris, 1966. 